\title{
The eclipsing binary IU Per and its intrinsic oscillations
}

\author{
X. B. Zhang $\dagger$ \\ National Astronomical Observatories, Chinese Academy of Sciences, Beijing, 100012, China \\ email: xzhang@bao.ac.cn;
}

\begin{abstract}
The results of a long-term time-series photometry of the short-period eclipsing binary IU Per are reported. The observation confirms the intrinsic $\delta$ Scuti-like pulsation of the star as discovered by previous authors. A photometric solution for the binary system was carried out with the new data. Based on which, the pure oscillation light variations from the mass-accreting primary component were extracted. A Fourier analysis reveals four pulsation modes. Combining with the photometric solution, a preliminary mode identification was given.
\end{abstract}

Keywords. binaries: eclipsing - stars: oscillations - stars: individual: IU Per.

\section{Introduction}

Pulsating stars in eclipsing binary systems are of peculiar interests for the theoretical study of stellar structure and evolution since binarity of these stars provides more useful information of the components. The existence of stellar pulsation in eclipsing binary systems has been noted early in 1970s. However, there are only about 40 of such samples have been found up to date.

The less-studied eclipsing binary IU Per is very probable a new sample consisting of a d Scuti-like pulsating component as reported by Kim et al. (2005). With a period of 0.8570257 day (Kreiner, 2004), it is one of the pulsating eclipsing system with the shortest orbital among all the samples. To check the pulsation nature of the binary system, we have observed the star photometrically for long time. It presents here the preliminary results of the observations.

\section{Observations}

All the observations were carried out at the Xinglong Station of NAOC. The fast photometry in 2006 was made with a 4-channel photoelectric photometor mounted on the $85-\mathrm{cm}$ reflector. A single narrow band b filter was used. Good data were collected on 3 nights. The V-band CCD photometry of IU Per was performed from Nov.03 to 15, 2007 by using the APM $50 \mathrm{~cm}$ telescope. The data were taken with a $1320 ? 1320$ CCD camera which provides a field size of 20.2 arcmin with a scale of 0.92 arcsec/pixel.. Exposure times were set from 10 to 15 seconds according to the weather conditions. Useful data were collected on 9 nights. A total number of 8400 frames were obtained. Adopting two stars, GSC0008-743 and GSC0008-949, as comparison and check stars, respectively, the differential photometry was extracted. The photometry precision is generally better than 0.005 mag. Fig. 1 presents an example of the time-series photometry. Inspecting the light curves. The short-term light variations in addition to the eclipsing light changes can be clearly seen. It confirms the discovery of Kim et al. (2005)

$\dagger$ Present address: National Astronomical Observatories., 20A Datun Road, Beijing, 10012, China. 
Table 1. Results of the Fourier analysis

\begin{tabular}{cccccc}
\hline$f_{i}$ & $\begin{array}{c}\text { Frequency } \\
(\mathrm{c} / \mathrm{d})\end{array}$ & $\begin{array}{c}\text { Amplitude } \\
(\mathrm{mmag})\end{array}$ & $\mathrm{S} / \mathrm{N}$ & $\begin{array}{c}\mathrm{Q} \\
(\times 100)\end{array}$ & Mode \\
\hline$f_{1}$ & $43.1314 \pm 0.0011$ & $3.08 \pm 0.07$ & 11.26 & 1.194 & $6 \mathrm{H}$ \\
$f_{2}$ & $47.7959 \pm 0.0017$ & $1.94 \pm 0.07$ & 8.16 & & $3 \times \mathrm{F}$ \\
$f_{3}$ & $15.9031 \pm 0.0031$ & $1.06 \pm 0.07$ & 3.81 & 3.239 & $\mathrm{~F}$ \\
$f_{4}$ & $40.9849 \pm 0.0036$ & $0.98 \pm 0.07$ & 3.23 & 1.257 & $5 \mathrm{H}$ \\
\hline
\end{tabular}
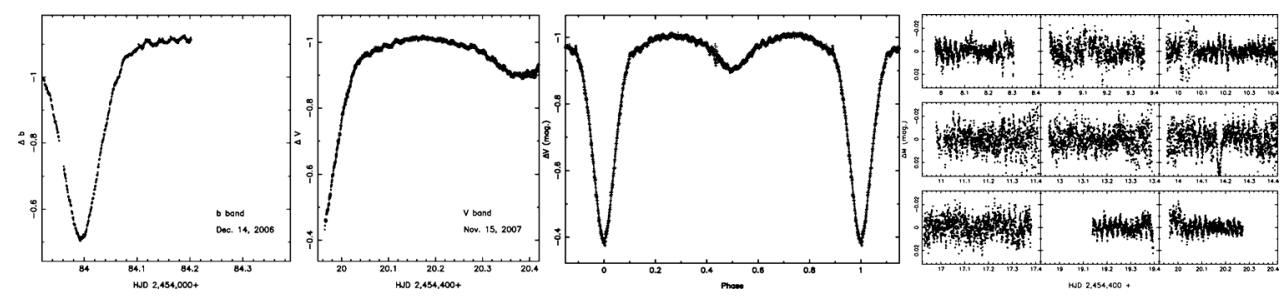

Figure 1. a, Real-time light curves of IU Per; b, The phased V light curve along with the theoretical fittings; c, The pulsating light variations extracted from the CCD data

\section{Light curve analysis}

A total of 7 new epoches of light minima were detected. With which, the orbital period of the system was renewed as 0.857025 days. The phased V-band light curve was shown in Fig. 1 along with a theoretical fitting based on a photometric solution by using the 2003 version of the $\mathrm{W}-\mathrm{D}$ code. The light curve synthesis yields a semi-detached configuration for the system with the secondary filling its own Roche lobe. The mass ratio of the system is derived to be about 0.27. According to the behavior of light variations at the eclipses, it is identified that the intrinsic oscillations are very probably from the mass-accreting primary component.

Based on the photometric solution, the theoretical time-series light curves for each star were computed. Following $l_{\text {obs }}=l_{1} f_{\text {pul }} .+l_{2}$, the intrinsic pulsation light variations from the primary star were then extracted as shown also in Fig. 1. A power spectral analysis was performed with the code Period04 to study the pulsation nature. It reveals four reliable pulsating frequenciess. The results are given in Table 1 . From the results of photometric solution, the mean density of the primary star was computed as $\rho_{1} / \rho_{\odot}=0.265$. With this value, a prilimary mode identitication was given.

\section{Conclusions}

The $\delta$ Scuti-like intrinsic oscillations of the eclipsing binary IU Per were confirmed. The star was proved to be a semi-detached algol system. With a new method, the intrinsic oscillation light variations from the mass-accreting component were extracted and the pulsating modes were identified. It suggests that IU Per could be a new member of the oscillating EA stars of peculiar interest for its short orbital period and the multi-periodic oscillations.

\section{Reference}

Kim, S. L., Lee, J. W., Koo, J. R., Kang, Y. B., \& Mkrtichian, D. E., 2005, IBVS, No. 5629 